Marquette University

e-Publications@Marquette

Physics Faculty Research and Publications

Physics, Department of

$2-1-2014$

Electron Cooling in a Young Radio Supernova: SN 2012aw

Naveen Yadav

Tata Instityute of Fundamental Research

Alak Ray

Tata Institute of Fundamental Research

Christopher Stockdale

Marquette University, christopher.stockdale@marquette.edu

Poonam Chandrasiri

National Center for Radio Astronomy-TIFR

Randall Smith

Harvard-Smithsonian Center for Astrophysics

See next page for additional authors

Published Version. Astrophysical Journal, Vol. 728, No. 1 (February 2014): 30-40. DOI: 10.1088/

0004-637X/782/1/30. (C) 2014 IOP Publishing. Used with permission. 
Authors

Naveen Yadav, Alak Ray, Christopher Stockdale, Poonam Chandrasiri, Randall Smith, Rupak Roy, Vikram Dwarkadas, Firoza Sutaria, and David Pooley 


\title{
ELECTRON COOLING IN A YOUNG RADIO SUPERNOVA: SN 2012aw
}

\author{
Naveen Yadav ${ }^{1}$, Alak Ray $^{1}$, Sayan Chakraborti $^{2}$, Christopher Stockdale $^{3}$, \\ Poonam Chandra ${ }^{4}$, Randall Smith ${ }^{5}$, Rupak Roy ${ }^{6}$, Subhash Bose ${ }^{6}$, Vikram Dwarkadas $^{7}$, \\ Firoza Sutaria ${ }^{8}$, AND David PoOley ${ }^{9}$ \\ ${ }^{1}$ Tata Institute of Fundamental Research, Homi Bhabha Road, Mumbai 400005, India; nyadav@tifr.res.in, akr@tifr.res.in \\ ${ }^{2}$ Institute for Theory and Computation, Harvard Smithsonian Center for Astrophysics, 60 Garden Street, Cambridge, MA 02138, USA \\ ${ }^{3}$ Marquette University, Milwaukee, WI 53233, USA \\ ${ }^{4}$ National Center for Radio Astronomy-TIFR, Pune 411007, India \\ ${ }^{5}$ Harvard-Smithsonian Center for Astrophysics, 60 Garden Street, Cambridge, MA 02138, USA \\ ${ }^{6}$ Aryabhhata Research Institute of Observational Sciences, Nainital 263129, India \\ ${ }^{7}$ Department of Astronomy \& Astrophysics, University of Chicago, Chicago, IL 60637, USA \\ ${ }^{8}$ Indian Institute of Astrophysics, Bangalore 560034, India \\ ${ }^{9}$ Department of Physics, Sam Houston State University, Huntsville, TX 77341, USA \\ Received 2013 August 1; accepted 2013 December 16; published 2014 January 23
}

\begin{abstract}
We present the radio observations and modeling of an optically bright Type II-P supernova (SN), SN 2012aw which exploded in the nearby galaxy Messier 95 (M95) at a distance of $10 \mathrm{Mpc}$. The spectral index values calculated using $C, X$, and $K$ bands are smaller than the expected values for the optically thin regime. During this time, the optical bolometric light curve stays in the plateau phase. We interpret the low spectral-index values to be a result of electron cooling. On the basis of comparison between the Compton cooling timescale and the synchrotron cooling timescale, we find that the inverse Compton cooling process dominates over the synchrotron cooling process. We therefore model the radio emission as synchrotron emission from a relativistic electron population with a high energy cutoff. The cutoff is determined by comparing the electron cooling timescale, $t_{\text {cool }}$, and the acceleration timescale, $\tilde{t}_{\text {acc }}$. We constrain the mass-loss rate in the wind $\left(\dot{M} \sim 1.9 \times 10^{-6} M_{\odot} \mathrm{yr}^{-1}\right)$ and the equipartition factor between relativistic electrons and the magnetic field $\left(\tilde{\alpha}=\epsilon_{e} / \epsilon_{B} \sim 1.12 \times 10^{2}\right)$ through our modeling of radio emission. Although the time of explosion is fairly well constrained by optical observations within about two days, we explore the effect of varying the time of explosion to best fit the radio light curves. The best fit is obtained for the explosion date as 2012 March 15.3 UT.
\end{abstract}

Key words: radiation mechanisms: non-thermal - radio continuum: general - stars: mass-loss - supernovae: individual (SN 2012aw) - techniques: interferometric - X-rays: general

Online-only material: color figure

\section{INTRODUCTION}

Core-collapse supernovae ( $\mathrm{SNe}$ ) mark the death of massive stars $\left(M_{*} / M_{\odot} \gtrsim 8\right)$. Study of electromagnetic emission from an SN across various wavelengths provides us with important clues about the nature of the explosion as well as the progenitor star. Early-time optical emission from an SN is used to derive many important parameters of the explosion (e.g., total explosion energy, nickel mass, etc.), whereas latetime optical emission is a probe of the inner layers of ejecta. Though not all $\mathrm{SNe}$ are detectable at radio wavelengths at a very young age, a small fraction of them are. According to the current understanding, this radio emission is nonthermal in origin (Chevalier 1982). The fast-moving SN ejecta drives a strong shock into the circumstellar medium (forward shock). Electrons are accelerated to relativistic energies at this shock. These electrons gyrate around the post-shock magnetic field and radiate via synchrotron emission. This radiation is an important probe of the pre-explosion evolution of massive stars.

During their evolution, massive stars lose mass (by either continuous stellar winds or periods of rapid/episodic mass loss; Dopita et al. 1984), which forms the circumstellar medium (CSM) in which the SN shock evolves. The velocity of stellar winds is small (for Wolf-Rayet stars it can be $20 \%$ of the ejecta velocity) compared to that of the $\mathrm{SN}$ ejecta, and therefore in a short time the fast moving ejecta probes a long period of mass loss. Observationally determined mass-loss rates can be used to constrain stellar evolution models. Young, radio-bright SNe also offer an opportunity to study particle acceleration and magnetic field amplification at these shocks.

Type II-P SNe are a class of core-collapse SN displaying an intermediate plateau phase in their bolometric light curve which extends from 60 to 100 days. They show a wide range of magnitude in both the plateau phase and expansion velocity (Hamuy 2003). Their progenitor stars have an extended hydrogen envelope prior to collapse (Smartt 2009). Therefore they are at the extremity of a range of stars retaining different hydrogen envelope masses at the time of explosion. As a result of the SN explosion, the hydrogen envelope is ejected at high velocity. The plateau phase is powered by a hydrogenrecombination wave traveling inward as this ejecta cools due to expansion and radiation losses. The photosphere demarcates this expanding hydrogen envelope into an inner region of high opacity and an outer region of low opacity. The plateau phase has been modeled numerically (Litvinova \& Nadezhin 1983; Bersten et al. 2011), semi-analytically (Falk \& Arnett 1977), and analytically (Arnett 1980; Chugai 1991; Popov 1993). The extended duration of the plateau phase makes these $\mathrm{SNe}$ more easily detectable even in low-cadence surveys. The long duration of the plateau phase may have consequences for the nonthermal radiation processes. The high radiation density of optical (UBVRI) photons during the plateau phase may cause effective cooling of relativistic electron population at the forward shock (Chevalier et al. 2006). 
X-ray emission from a young Type II-P SN can be thermal or nonthermal in origin (Chevalier 1982). The thermal component can originate as a result of free-free emission in the post-shock region or at the reverse shock (a shock which is driven in to the expanding SN ejecta), whereas the nonthermal emission can be due to inverse Compton scattering of low-energy photons by relativistic electrons at the forward shock. Therefore, in case an SN is bright and detectable in X-rays at early times, much more information is available for understanding the dynamics of the forward shock, the reverse shock, the density profile of the ejecta, and the circumstellar medium. In the case of SN 2004dj, Chakraborti et al. (2012) have estimated various important parameters relevant to blast-wave dynamics and particle acceleration using four epochs of Chandra observations. In the case of SN 2011 ja, Chakraborti et al. (2013) have reported that the X-ray flux from this SN during the second observation epoch was higher compared to the X-ray flux during the first epoch by a factor of 4.2. They have argued that it can be explained by an enhancement in the density of the circumstellar medium probed by the shock at a later time and have suggested that a fraction of Type II-P explosions may take place inside bubbles blown by hot winds or a variable circumstellar medium created by nonsteady winds. Therefore, following the temporal evolution of young Type II-P explosions in radio and X-ray bands will provide us with crucial information about both the explosions and the surrounding media created during the late evolution of their progenitor stars.

SN 2012aw is a bright Type II-P SN which exploded in the galaxy M95 ( $d \sim 10 \mathrm{Mpc}$ ). Spectra taken four to five days after discovery showed it to be a Type II-P explosion (Fagotti et al. 2012). Fraser et al. (2012) identified a candidate progenitor star in archival Hubble Space Telescope images. Fraser et al. (2012) have inferred a progenitor mass in the range 14-26 $M_{\odot}$, whereas Van Dyk et al. (2012) inferred a progenitor mass in the range $17-18 M_{\odot}$. The progenitor seems to be a faint, red supergiant and is the most massive Type II-P progenitor discovered to date. Both works noted that the star had a significantly higher extinction prior to its explosion as an SN and interpret it as a signature of dust destruction by explosion. Fraser et al. (2012) noted that the progenitor's luminosity is not very well constrained because of uncertainty in the extinction, which will further affect the estimates on the progenitor's mass. Van Dyk et al. (2012) claimed evidence for dust destruction by explosion, as the current extinction to the $\mathrm{SN}$ is very low. This may have interesting consequences for the progenitors of Type II-P SN. SN 2012aw has been extensively studied through optical and UV photometry. Bose et al. (2013) have found that SN 2012aw has remarkable similarities with $\mathrm{SNe}$ 1999em, 1999gi, and 2004et. Bose et al. (2013) have reported nebular spectroscopy of the SN at an age of 270 days and, on the basis of lines profile shapes, claimed that there are no signs of fresh dust formation. Immler \& Brown (2012) reported the detection of an X-ray point source consistent with the optical position of SN 2012aw, with a 3.8 $\sigma$ significance. We triggered the $K$-band radio observation of SN 2012aw under our Joint Chandra-EVLA proposal (Proposal No. 13500809) to observe bright and nearby Type II-P events. After the initial detection (Yadav et al. 2012), the JVLA radio follow-up was carried out through Jansky VLA Director's Discretionary Time. We have observed the object at radio wavelengths using $\mathrm{JVLA}^{10}$

\footnotetext{
10 The National Radio Astronomy Observatory is a facility of the National Science Foundation operated under cooperative agreement by Associated Universities, Inc.
}

and GMRT, ${ }^{11}$ targeting it at $L(1.4 \mathrm{GHz}), S(3.0 \mathrm{GHz}), C$ $(5.0 \mathrm{GHz}), X(8.5 \mathrm{GHz}), K(21.0 \mathrm{GHz})$, and $K a(32.0 \mathrm{GHz})$ bands at multiple epochs. In this work, we present the analysis and modeling of radio observations of this SN. We model the radio observations using the circumstellar interaction model. We show that there is a signature of electron cooling in the spectral evolution of the SN, especially at high frequencies. In our model, we modify the electron population by taking inverse Compton cooling processes in to consideration. We constrain the parameters relevant to the progenitor (mass-loss rate, $\dot{M} / v_{w}$, where $v_{w}$ is the wind velocity) and properties of shock acceleration (equipartition factor, $\epsilon_{e} / \epsilon_{B}$, where $\epsilon_{e}$ and $\epsilon_{B}$ are the fractions of energy in relativistic electrons and the post-shock magnetic field, respectively).

\section{RADIO OBSERVATIONS \& REDUCTION}

SN 2012aw was first detected in the radio JVLA- $K$ band (21 GHz) at $\sim 10$ days by Yadav et al. (2012) and Stockdale et al. (2012). We conducted the follow-up radio observations of 2012aw at various epochs extending up to 184 days after the explosion using the Karl G. Jansky Very Large Array (JVLA) and the Giant Meterwave Radio Telescope (GMRT). These observations have been reduced using Astronomical Image Processing Software (AIPS) standard techniques. Group-delay and phaserates calibration were determined using the AIPS task FRING. Noisy data were flagged and the interferometric visibilities have been calibrated using 3C286. Bandpass calibration was done using BPASS based on the strong flux calibrators. The singlesource data have been extracted using the AIPS task SPLIT after final calibration. The single-source data were imaged using IMAGR. The images were corrected for residual calibration errors using self-calibration of visibility phases (Cornwell \& Fomalont 1989). The source fluxes were extracted by fitting Gaussians using the task JMFIT and assuming point sources. The errors reported on the flux are obtained by using the image statistics from the region surrounding the source.

In the case of SN 2012aw, the explosion date is strongly constrained to within \pm 1.6 days based on a nondetection (limiting magnitude of $R \gtrsim 20.7$ ) on March 15.27 reported by Poznanski et al. (2012) and the first detection on March 16.9 reported by Fagotti et al. (2012). We have used the explosion date as March 16.1 in this work. We have explored the effect of varying the explosion date within the 1.6 day time range. The radio observations are presented in Table 1.

\section{MODELING THE RADIO OBSERVATIONS}

The interaction of fast-moving ejecta with the circumstellar medium drives a strong shock that moves ahead of the ejecta into the circumstellar medium and is called the "forward shock." Electrons are accelerated to relativistic energies at this shock via the Fermi first-order process. These electrons radiate via the synchrotron mechanism in the post-shock magnetic field. The electron spectrum is described as

$$
N(E)=N_{0} E^{-\gamma}
$$

where $N_{0}$ is the normalization constant and $\gamma$ is the electron index. The radio emission from young $\mathrm{SNe}$ is generally modeled

\footnotetext{
11 We thank the staff of the GMRT that made these observations possible. GMRT is run by the National Center for Radio Astrophysics of the Tata Institute of Fundamental Research.
} 
Table 1

Radio Observations of SN 2012aw

\begin{tabular}{|c|c|c|c|c|}
\hline $\begin{array}{l}\text { Date } \\
\text { (UT) }\end{array}$ & $\begin{array}{c}\Delta t \\
\text { (days) }\end{array}$ & $\begin{array}{c}v \\
(\mathrm{GHz})\end{array}$ & $\begin{array}{c}f_{v} \\
(\mu \mathrm{Jy})\end{array}$ & $\begin{array}{c}\mathrm{rms} \\
(\mu \mathrm{Jy})\end{array}$ \\
\hline Mar24.10 & 8.00 & 8.5 & 76 & 12.0 \\
\hline Mar24.10 & 8.00 & 20.8 & 160 & 25.0 \\
\hline Mar30.09 & 14.00 & 21.2 & 310 & 19.6 \\
\hline Apr01.31 & 16.00 & 20.8 & 220 & 38.0 \\
\hline Apr03.08 & 17.98 & 8.5 & 474 & 11.0 \\
\hline Apr03.08 & 17.98 & 5.0 & 327 & 11.0 \\
\hline Apr08.04 & 22.94 & 8.9 & 510 & 27.4 \\
\hline Apr09.04 & 23.94 & 5.5 & 559 & 42.7 \\
\hline Apr09.04 & 23.94 & 2.9 & 264 & 45.4 \\
\hline Apr09.04 & 23.94 & 1.5 & $<150.0$ & 50.0 \\
\hline Apr14.19 & 29.09 & 21.2 & 157 & 12.2 \\
\hline Apr16.02 & 30.92 & 3.0 & 219 & 37.6 \\
\hline Apr16.02 & 30.92 & 5.5 & 572 & 23.1 \\
\hline Apr16.02 & 30.92 & 8.9 & 468 & 27.8 \\
\hline Apr18.12 & 33.02 & 32.0 & 110 & 19.5 \\
\hline Apr22.05 & 36.95 & 3.1 & 554 & 78.4 \\
\hline Apr22.05 & 36.95 & 5.5 & 493 & 19.2 \\
\hline Apr22.05 & 36.95 & 9.0 & 299 & 27.6 \\
\hline Apr24.27 & 39.17 & 21.2 & 142 & 19.1 \\
\hline May08.06 & 52.94 & 3.0 & 707 & 72.8 \\
\hline May08.06 & 52.94 & 5.5 & 554 & 17.6 \\
\hline May08.06 & 52.94 & 9.0 & 293 & 19.5 \\
\hline May08.06 & 52.94 & 21.2 & 96 & 9.2 \\
\hline May19.10 & 64.00 & 3.2 & 560 & 53.6 \\
\hline May19.10 & 64.00 & 5.5 & 421 & 17.3 \\
\hline May19.10 & 64.00 & 9.0 & 267 & 17.7 \\
\hline May19.10 & 64.00 & 21.2 & 103 & 21.6 \\
\hline May23.19 & 68.09 & 32.0 & $<60.0(4 \sigma)$ & 15.0 \\
\hline Jun27.10 & 103.00 & 3.2 & 347 & 22.2 \\
\hline Jun27.10 & 103.00 & 5.5 & 245 & 15.9 \\
\hline Jun27.10 & 103.00 & 9.0 & 154 & 13.9 \\
\hline Jul28.03 & 133.97 & 3.2 & 343 & 25.0 \\
\hline Jul28.03 & 133.97 & 5.5 & 220 & 18.9 \\
\hline Jul28.03 & 133.97 & 8.9 & 126 & 14.2 \\
\hline Sep15.00 ${ }^{\mathrm{a}}$ & 182.90 & 1.3 & 436 & 81.0 \\
\hline
\end{tabular}

Notes. The explosion date is taken to be 2012 March 16.1(UT).

a GMRT data point.

as synchrotron emission by this electron population affected by a variety of absorption processes. The absorption can be modeled as a combination of synchrotron self absorption (SSA; the electrons that are responsible for synchrotron emission also absorb the synchrotron photons) and free-free absorption (FFA; the thermal electrons in the post-shock medium absorb the synchrotron photons). We use Chevalier model-I (Table 1, Chevalier 1996) to study this emission. In this model, the radius of the forward shock increases as $R \propto t^{m}$, and the energy densities in both relativistic electrons and the magnetic field are proportional to the thermal energy density, which leads to $u_{e}, u_{B} \propto t^{-2}$, where $u_{e}$ is the energy density in the relativistic electrons and $u_{B}$ is the energy density in the post-shock magnetic field, respectively. Another important assumption inherent to the model is that the electron index $\gamma$ remains constant during the evolution. The electron index can be obtained by fitting a power law to the optically thin component. The equation for the radio-flux evolution in such a case is given in Chevalier (1998) for the case of an $\mathrm{SN}$ blast wave expanding into a circumstellar medium set up by a uniform wind $\left(\rho_{w} \propto r^{-2}\right)$. If we try to model the radio emission from SN 2012aw by a simple SSA+FFA model, the best fit gives $\chi_{v}^{2} \sim 7.2$, but results in a value of $m$ greater than 1 (SSA model: $m=1.1 \pm 0.02$; SSA+FFA model:

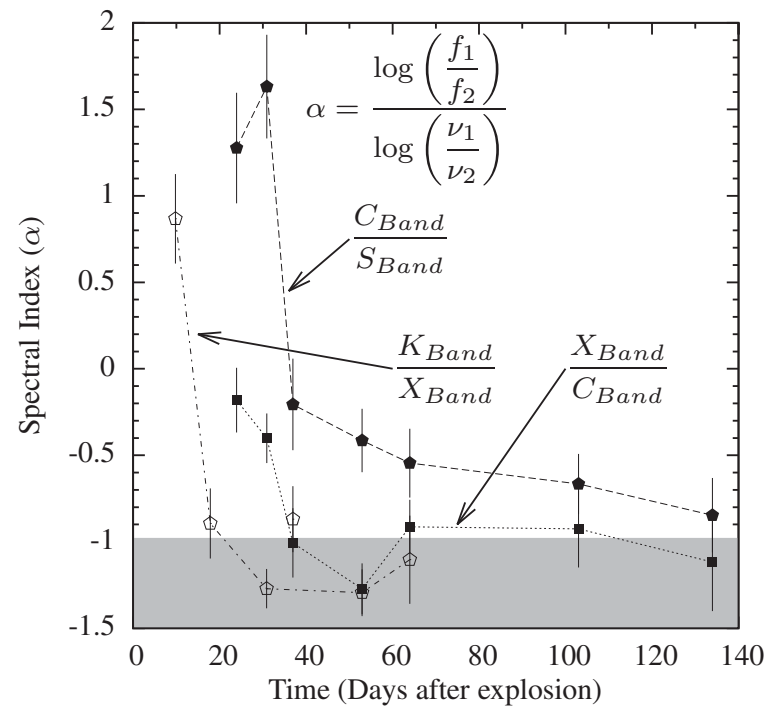

Figure 1. Spectral-index curves made using the $S$-, $C$-, $X$-, and $K$-band data. In case of the $X_{\text {Band }} / C_{\text {Band }}$ and $K_{\text {Band }} / X_{\text {Band }}$ ratios, the spectral-index values are smaller than -1 for an extended period of time between 20 and 60 days. The $C_{\text {Band }} / S_{\text {Band }}$ index approaches the optically thin value slowly as the SN ages. The spectral indices calculated from higher frequency bands show observable deviations due to cooling of the relativistic electrons.

$m=1.08 \pm 0.02$ ), implying an accelerated blast wave, which is unlikely as the blast wave decelerates due to its interaction with the circumstellar matter. The difference between the model and the data at early times is relatively large compared to that at late times.

In order to explore the nature of the electron cooling processes further, we make a study of the spectral-index evolution using our radio data as shown in Figure 1. In the case when a source that can be described by a simple SSA+FFA model without cooling, the radio spectral index approaches the value $-(\gamma-$ 1) $/ 2$ as the source enters the optically thin regime. The spectralindex curves labeled as " $X_{\text {Band }} / C_{\text {Band }}$ " and " $K_{\text {Band }} / X_{\text {Band }}$ " have values lower than -1 for an extended period of time, during which the $\mathrm{SN}$ has a plateau in its optical bolometric light curve, whereas the " $C_{\text {Band }} / S_{\text {Band" }}$ " spectral-index values slowly approach the optically-thin-regime value. This is because, due to electron cooling, the flux in the higher frequency bands is diminished more in comparison to lower frequency bands, and this leads to a dip in the spectral index. The simplistic model proposed here may not fully account for the dip in the spectralindex curves, indicating that one may need to go beyond the simple model described here to accommodate early-time, highfrequency observations. A more realistic model will include the effect of variation in both electron index and mass loss, as has been done in the case of SN 1993J by Fransson \& Björnsson (1998).

Electron cooling can be due to Coulomb, synchrotron, or inverse Compton mechanisms or adiabatic expansion. Cooling has been discussed in the case of Type II-P SNe by Chevalier et al. (2006), and Björnsson \& Fransson (2004) have discussed its importance in the case of SN 2002ap, a Type Ic event. To determine the dominant cooling mechanism, we need to compare the cooling timescales for various mechanisms.

\section{COOLING TIMESCALES}

The rates at which an electron of energy $E$ loses energy by adiabatic expansion, inverse Compton scattering, and synchrotron 
emission are $^{12}$

$$
\begin{aligned}
& \left(\frac{d E}{d t}\right)_{\mathrm{AD}} \approx \frac{2}{3} E t, \\
& \left(\frac{d E}{d t}\right)_{\mathrm{IC}} \propto u_{\mathrm{rad}} E^{2}, \\
& \left(\frac{d E}{d t}\right)_{\mathrm{SC}} \propto B^{2} E^{2},
\end{aligned}
$$

respectively, where $u_{\text {rad }}$ is the energy density of the radiation field and $B$ is the magnetic field. The characteristic energyloss timescale $t$ can be written as $E / \dot{E}$. The adiabatic-cooling timescale is $t_{\mathrm{ad}} \propto t$ (Chevalier 1982). The cooling timescales for inverse Compton and synchrotron emission can therefore be written using the formulae for energy loss from Pacholczyk (1970) as

$$
\begin{aligned}
t_{\mathrm{IC}} & =\frac{1}{3.97 \times 10^{-2} u_{\mathrm{rad}} E}, \\
t_{\mathrm{SC}} & =\frac{1}{5.95 \times 10^{-2} u_{B} E},
\end{aligned}
$$

where $u_{\text {rad }}$ in our case is the energy density of photons at the $\mathrm{SN}$ radiosphere and $u_{B}$ is the energy density of the post-shock magnetic field. In the following subsections, we will compare the cooling timescale for inverse Compton and synchrotron loses and determine the dominant cooling mechanism. In order to compare the cooling timescales, we first need to estimate the post-shock magnetic field and the radiation density at the forward shock.

\subsection{Post-shock Magnetic Field}

To get the synchrotron-cooling timescale, we need an estimate of the magnetic field. In the CSM interaction models for radio $\mathrm{SNe}$, the post-shock magnetic field is assumed to scale with time according to a power law. In Chevalier model-I, the magnetic field evolves as $t^{-1}$. This is because the magnetic energy density is proportional to the thermal energy density, which for a constant-parameter wind medium goes as $t^{-2}$, therefore $B \propto t^{-1}$. If we know the magnetic field at epoch $t_{0}$, it can simply be scaled to get the field at any other epoch using

$$
B(t)=B_{0}\left(\frac{t}{t_{0}}\right)^{a} .
$$

We use the value $a=-1$ in our calculations, in accordance with Chevalier model-I. To get an estimate of the magnetic field, we can either use a late-time radio spectrum or a low-frequency radio light curve, which are relatively free from the electroncooling effects. We consider the $3 \mathrm{GHz}$ light curve for this part of calculation. In order to have minimum free parameters, we need to check whether FFA is important to model the $3 \mathrm{GHz}$ radio data available to us ( $t>23$ days).

To get an estimate of the FFA,we use $\dot{M}_{-5} / v_{w 1}$ determined from the epoch of X-ray detection (time at which the optical depth to X-rays becomes unity), where $\dot{M}_{-5}$ is the mass-loss rate in units of $10^{-5} M_{\odot} \mathrm{yr}^{-1}$, and $v_{w 1}$ is the wind velocity

\footnotetext{
12 Details of the energy-loss formula are given in Rybicki \& Lightman (1986)
} and, for the case of a supernova (SN 1993J), by Fransson \& Björnsson (1998).

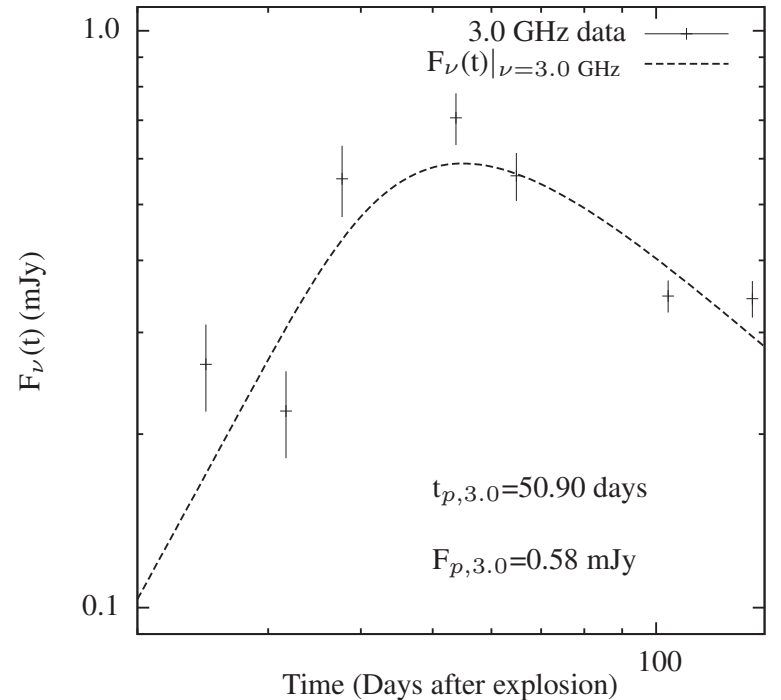

Figure 2. SSA model fit to the $3.0 \mathrm{GHz}$ radio light curve using an electron index of $\gamma=3.1$. The radio light curve in $3.0 \mathrm{GHz}$ band peaks at 50.90 days with the peak flux density of $0.58 \mathrm{mJy}$ and $m=0.97$. The $\chi_{v}^{2} \sim 5.4$ for the fit.

in units of $10 \mathrm{~km} \mathrm{~s}^{-1}$. This object was first detected in X-rays (0.2-10 keV band) by Immler \& Brown (2012) approximately 4 days after the explosion. This has been used to get an upper limit on the quantity $\dot{M}_{-5} / v_{w 1}$, which characterizes mass loss by a uniform wind. Using Equation (2.17) from Chevalier \& Fransson (1994), we get

$$
\frac{\dot{M}_{-5}}{v_{w 1}}=\frac{8.64 \times 10^{4} t_{X} v_{s 4} E_{\mathrm{KeV}}^{8 / 3}}{C_{5}},
$$

where $v_{s 4}$ is the outer (forward) shock velocity in units of $10^{4} \mathrm{~km} \mathrm{~s}^{-1}, t_{X}$ is the time at which the medium becomes optically thin to X-rays of energy $E_{\mathrm{KeV}}$, and $C_{5}$ is a constant. Using $t_{X}=4$ days, $v_{s 4} \sim 1.0$, and $E_{\mathrm{KeV}}=1.0$, Kochanek et al. (2012) find that there are no clear detections at low energies $(0.2-0.5 \mathrm{keV})$ and only marginal detections at high energies $(2-10 \mathrm{keV})$ - the observed counts are completely dominated by the $0.5-2 \mathrm{keV}$ band. Substituting the value $C_{5}=2.6 \times 10^{6}$ into Equation (8),

$$
\frac{\dot{M}_{-5}}{v_{w 1}}<0.13 \text {. }
$$

This is used to get an upper limit on the time for which FFA dominates at any radio frequency. Using Equation (4) from Chevalier et al. (2006),

$$
t_{\mathrm{ff}} \approx 6\left(\frac{\dot{M}_{-6}}{v_{w 1}}\right)^{2 / 3} T_{\mathrm{cs} 5}^{-1 / 2} v_{s 4}^{-1}\left(\frac{v}{8.46 \mathrm{GHz}}\right)^{-2 / 3}
$$

where $t_{\mathrm{ff}}$ is the time when the free-free opacity becomes low enough so that the medium becomes transparent to radio waves, and $T_{\mathrm{cs} 5}$ is the circumstellar temperature in units of $10^{5} \mathrm{~K}$. This gives $t_{\mathrm{ff}} \leqslant 16.0$ days at $3.0 \mathrm{GHz}$ and $t_{\mathrm{ff}} \leqslant 11.0$ days at $5.0 \mathrm{GHz}$ for $T_{\mathrm{cs} 5}=1.0$. This shows that the $3 \mathrm{GHz}$ radio light curve is not dominated by FFA in its optically thick phase (because our $3 \mathrm{GHz}$ radio observations start from 23 days after the explosion, whereas $t_{\mathrm{ff}}<16$ days). The $3.0 \mathrm{GHz}$ light curve can thus be fitted by a pure SSA model (Equation (4), Chevalier 1998) as shown in Figure 2. The fitted value of $m$ is found to be $\sim 0.97$ for the explosion date, 2012 March 15.3 UT. 

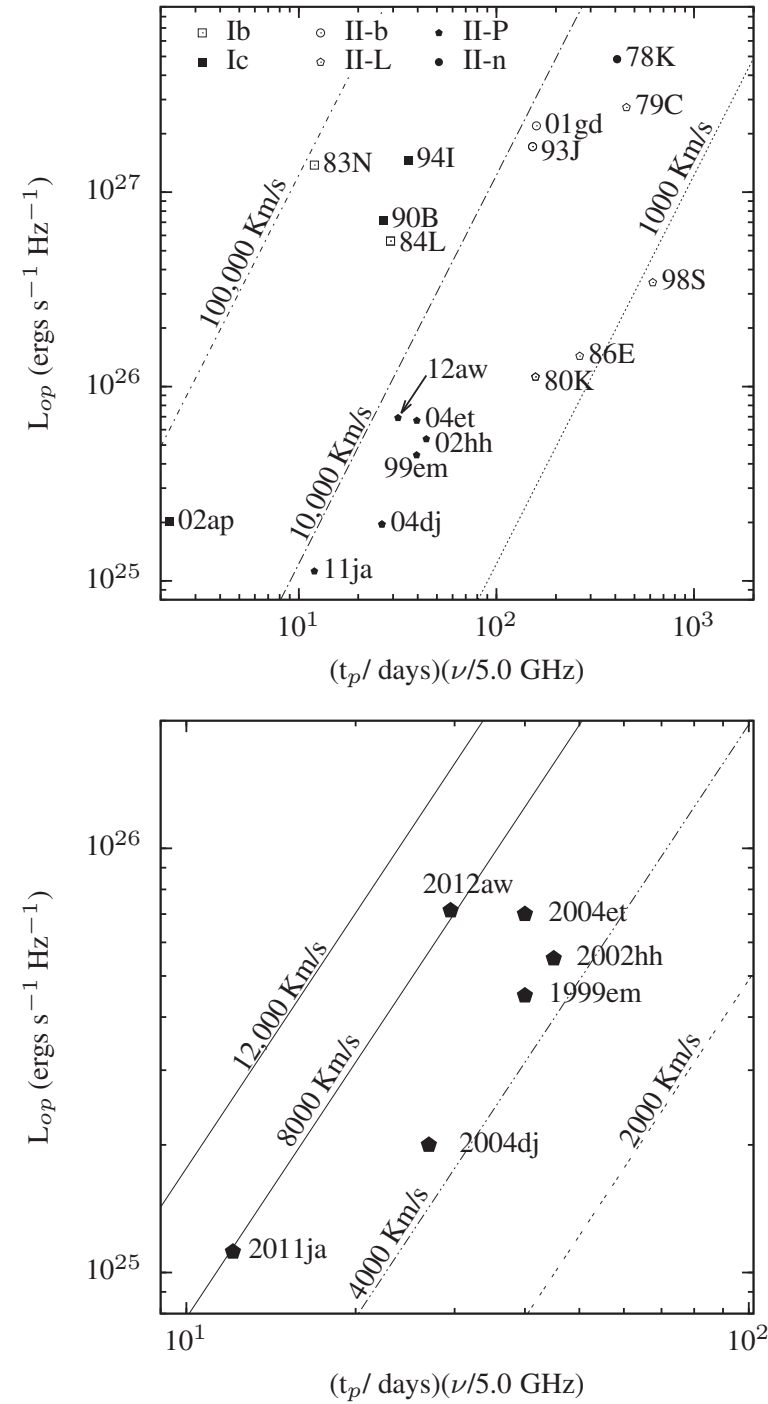

Figure 3. Upper panel: Type II-P SN shown in the $L_{o p}-v_{p} t_{p}$ plot (after Chevalier 1998) relative to other core collapse SNe. Lower panel: SN 2012aw placed on a magnified $L_{o p}-v_{p} t_{p}$ plot along with other radio Type II-P SNe. The constant velocity lines have been calculated using an electron index of $\gamma=3.0$.

A change in the assumed explosion date leads to differing values of the best fit $m$. The peak radio flux and the time to peak can be used to estimate the values of the radius and the magnetic field strength. Using Equations (11) and (12) from Chevalier (1998) gives $B_{0} \sim 0.48 \mathrm{G}$ and $R_{0} \sim 3.9 \times 10^{15} \mathrm{~cm}$ at an age of $\sim 50.9$ days, assuming equipartition. The magnetic field-assuming a different value of the equipartition factor $\left(\tilde{\alpha}=\epsilon_{e} / \epsilon_{B}\right) —$ can be written as

$$
\begin{gathered}
B_{0}(\tilde{\alpha})=0.46 \tilde{\alpha}^{-4 /(2 \gamma+13)} \mathrm{G}, \\
R_{0}(\tilde{\alpha})=4.9 \times 10^{15} \tilde{\alpha}^{-1 /(2 \gamma+13)} \mathrm{cm} .
\end{gathered}
$$

We can now put the object on an $L_{o p}-v_{p} t_{p}$ plot, as shown in Figure 3, to compare it with the known Type II-P SNe. The object has a higher expansion velocity among known radio bright SNe. The $L_{o p}$ and $v_{p} t_{p}$ values for SN $1999 \mathrm{em}, 2002 \mathrm{hh}$, 2004et, and 2004dj have been taken from Chevalier et al. (2006), and the values for SN 2011ja have been taken from Chakraborti et al. (2013). The plot has been generated for an electron index $\gamma=3$.0. SN 2012aw falls on a constant-velocity line at around

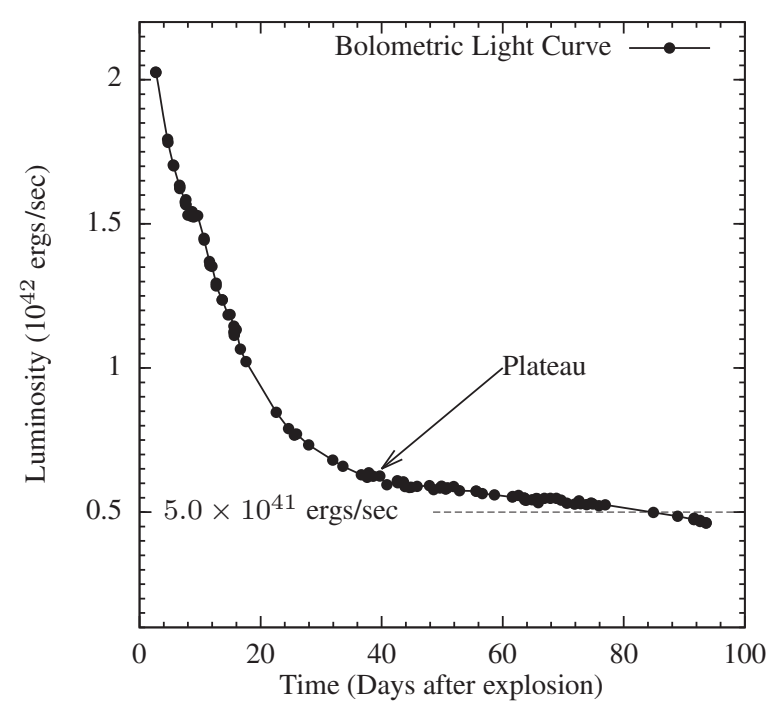

Figure 4. Bolometric light curve of SN 2012aw calculated using the published UBVRI photometry from Bayless et al. (2013) and Munari et al. (2013). The details of this procedure are described in Section 4.2. The value of the plateauphase luminosity is around $\sim 5.0 \times 10^{41} \mathrm{erg} \mathrm{s}^{-1}$.

$8.0 \times 10^{3} \mathrm{~km} \mathrm{~s}^{-1}$, which is a typical value of the blast-wave speed. It also shows that the object is not much affected by FFA, which is consistent with the low mass-loss rate suggested by the X-ray detection. The seemingly slow objects between the $4.0 \times 10^{3} \mathrm{~km} \mathrm{~s}^{-1}$ and $8.0 \times 10^{3} \mathrm{~km} \mathrm{~s}^{-1}$ lines are either dominated by FFA at early times or affected by cooling at early times.

\subsection{Radiation Density and Bolometric Light Curve}

To get the Compton-cooling timescale, we need the bolometric luminosity. We construct a bolometric light curve using published photometric (UBVRI) data from Bayless et al. (2013; Swift photometry) and Munari et al. (2013). We take the available photometric data and fill in the gaps using linear interpolation. Note that we have not included the infrared photometry, which is not available at these epochs; therefore the bolometric luminosity may be higher by at most $\sim 0.30$ dex during the plateau phase. The Swift photometry has been converted to flux from count rate using count-to-flux conversion factors from Poole et al. (2008). We calculate the bolometric light curve by integrating over the resulting photometric data using a simple trapezoidal integration rule. The calculated bolometric light curve is shown in Figure 4. The late part ( $t>200$ days) of the bolometric light curve used in the calculation is taken from Bose et al. (2013), who have also calculated the photospheric radius and temperature evolution of SN 2012aw. The radiation density at the radiosphere can be calculated from

$$
u_{\mathrm{rad}}(t)=\frac{L_{\mathrm{Bol}}(t)}{4 \pi R(t)^{2} c},
$$

where $R(t)$ is the radius of the radiosphere (forward shock) at a given time and is given by

$$
R(t)=R_{0}\left(\frac{t}{t_{0}}\right)^{m} .
$$

\subsection{Inverse Compton versus Synchrotron Cooling}

The calculated inverse-Compton and synchrotron cooling timescales for electrons of different Lorentz factors, $\gamma_{i}$, are shown in Figure 5 in comparison to the adiabatic timescale. 


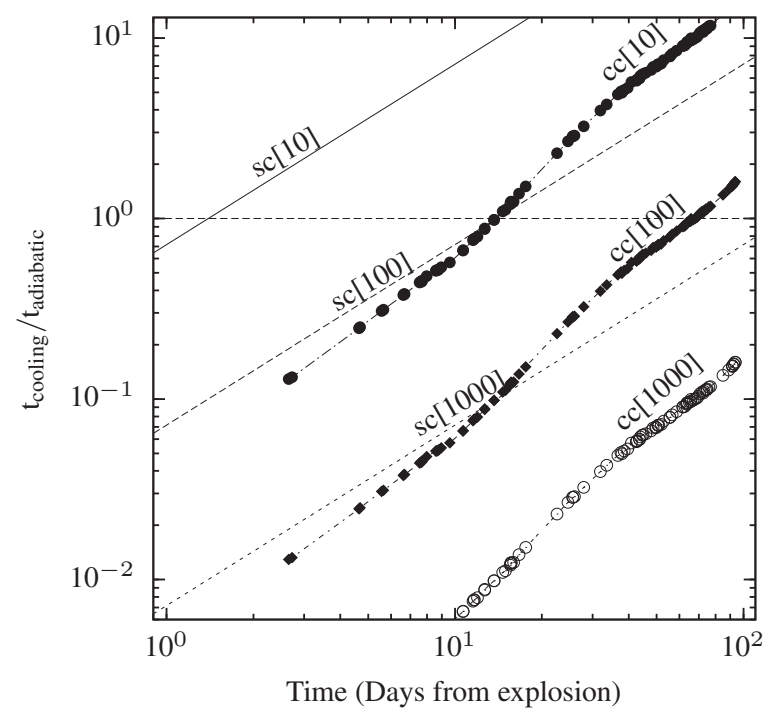

Figure 5. Cooling timescales for electrons calculated using the computed bolometric light curve for various values of Lorentz factors as marked in the diagram by $\mathrm{sc}\left[\gamma_{i}\right]$ for synchrotron Cooling and $\mathrm{cc}\left[\gamma_{i}\right]$ for Compton cooling, respectively. Note that the Compton cooling process is dominant over the synchrotron cooling process at any given Lorentz factor, $\gamma_{i}$. Also both inverse Compton cooling and synchrotron cooling dominate over the adiabatic cooling.

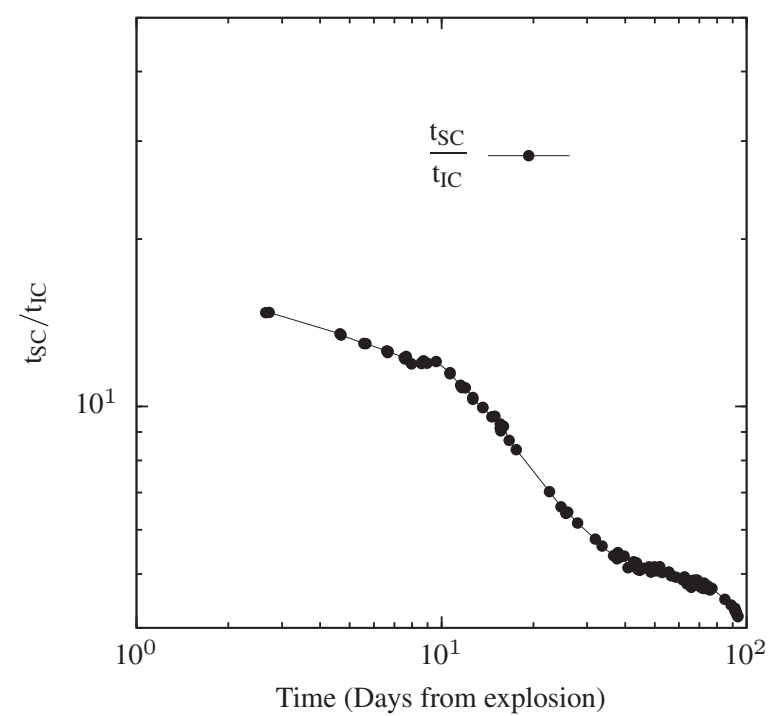

Figure 6. Ratio of synchrotron and Compton cooling timescales for electrons calculated using the computed bolometric light curve. Note that in the case of SN 2012aw, the Compton cooling process is dominant over the synchrotron cooling process because $t_{\text {Comp }} \ll t_{\text {Sync }}$. After around 100 days, the object enters the regime where synchrotron cooling is dominant over inverse Compton cooling, but by that age adiabatic expansion losses are the most important (see Figure 5).

At all values of $\gamma_{i}$, the inverse-Compton cooling timescale is very small compared to the synchrotron cooling timescale.

The ratio of synchrotron and Compton cooling timescales is independent of electron energy:

$$
\frac{t_{\mathrm{SC}}}{t_{\mathrm{IC}}} \propto \frac{u_{\mathrm{rad}}}{u_{B}} .
$$

It is evident from Figure 6 that Compton cooling dominates over the synchrotron cooling mechanism. Therefore, in order to model the radio spectrum at early epochs and at high frequency, we need to consider the effect of the inverse Compton cooling mechanism on emission. This can be done by modeling the

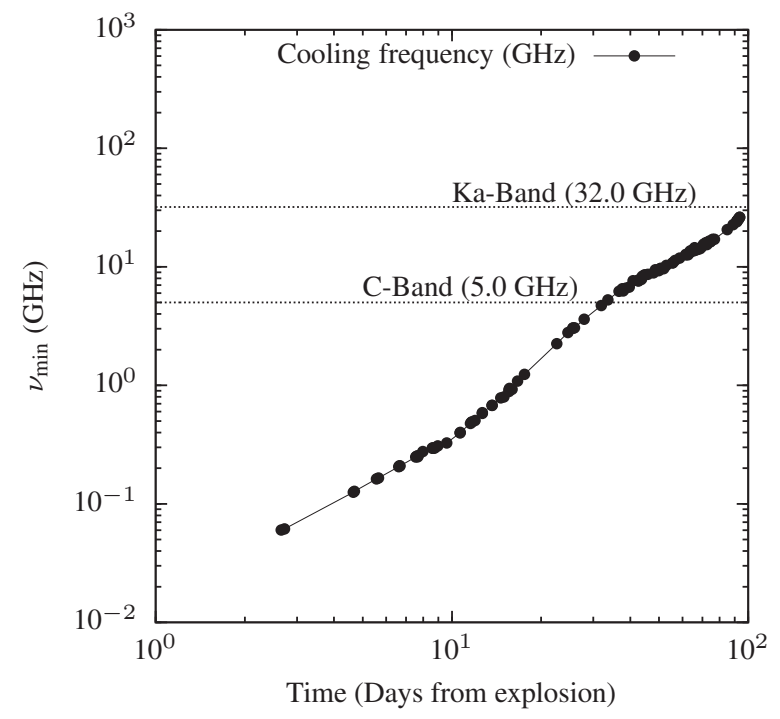

Figure 7. Minimum frequency affected by cooling plotted as a function of the age of SN 2012aw. Note that the dotted lines at 32.0 GHz and 5.0 GHz show that at early times all the VLA bands will have some effect from electron cooling. For the $C$-Band, the cooling phase lasts until $\sim 30$ days. The rate of energy loss by relativistic electrons due to Compton scattering is proportional to the radiation density. During the plateau phase of a Type II-P SN, the bolometric luminosity remains high for an extended period of time. This leads to electron cooling. The rate of cooling is proportional to the square of the electron energy, therefore the higher energy electrons cool much faster than the lower energy electrons. Therefore the higher frequency bands are affected relatively more compared to the lower frequency bands.

kinetic equation for electrons with the relevant energy-loss terms included.

\subsection{Cooling Frequency}

Assuming that an electron emits synchrotron radiation at its characteristic frequency, $v_{c}$, we can get an estimate of frequencies that are affected at a given age by comparing the adiabatic timescale, $t_{\mathrm{ad}} \sim 1.5 t$, and the Compton cooling timescale, $t_{\mathrm{IC}}$. Electrons that are affected by cooling $\left(t_{\mathrm{Comp}}<\right.$ $\left.t_{\mathrm{ad}}\right)$ have energies greater than

$$
E>\frac{1}{3.97 \times 10^{-2} u_{\mathrm{rad}} \times 1.5 t} .
$$

Using $v_{c} \sim c_{1} B E^{2}$, where $c_{1}$ is a constant, the minimum frequency above which effects due to Compton cooling are present can be written as

$$
\begin{aligned}
v_{\text {min }} & \gtrsim c_{1} \times B_{0}\left(\frac{t_{0}}{t}\right)\left(\frac{4 \pi R_{0}^{2} c t}{5.96 \times 10^{-2} t_{0}^{2} L_{\mathrm{bol}}}\right)^{2} \\
v_{\min } & =\frac{c_{1} B_{0}}{t_{0}^{3}} \times\left(\frac{4 \pi R_{0}^{2} c}{5.96 \times 10^{-2}}\right)^{2} \times\left(\frac{t}{L_{\mathrm{bol}}^{2}}\right) \\
& =0.78\left(\frac{t}{10 \text { days }}\right)\left(\frac{L_{\mathrm{Bol}}}{10^{42}}\right)^{-2} \mathrm{GHz} .
\end{aligned}
$$

The minimum frequency that is affected by cooling is shown in Figure 7. It shows that at very early times most of the JVLA radio bands are affected, but as the SN bolometric flux decreases, $v_{\min }$ goes to larger and larger values, as can be seen from Equation (19). It shows that electron cooling needs to be considered for a self-consistent modeling of early-time, highfrequency radio emission. 


\section{COOLING AFFECTED ELECTRON POPULATION}

In order to evaluate the effect of electron cooling on radio emission, we can solve the full electron kinetic equation numerically and calculate the fluxes at any given time from the resulting electron distribution. The rate of change of energy of an electron is given by

$$
\frac{d E}{d t}=\left(\frac{d E}{d t}\right)_{+}-\left(\frac{d E}{d t}\right)_{-},
$$

where "+" and "-" represent energy gain and loss processes. At an energy $E_{\max }$, both rates can become equal, and the electron cannot be accelerated further. We therefore obtain an electron distribution that is bounded at the higher energy end. The cutoff is dependent on the bolometric luminosity and the radius of the forward shock. We can get the upper limit on the energy by comparing the cooling timescale $t_{\text {Comp }}$ and the average acceleration timescale $\tilde{t}_{\mathrm{acc}}$ (it quantifies the time required for an electron to be accelerated to a given energy) for radio emitting electrons. The condition for $E_{\max }$ is

$$
\frac{t_{\text {Comp }}}{\tilde{t}_{\mathrm{acc}}}<1,
$$

The inequality gives $E_{\max }$ in terms of bolometric luminosity, forward shock radius, and $\tilde{t}_{\text {acc }}$ as a function of time:

$$
E_{\max }=\frac{4 \pi R(t)^{2} c}{3.97 \times 10^{-2} \tilde{t}_{\mathrm{acc}} L_{\mathrm{bol}}(t)} .
$$

We truncate the original power-law electron distribution at $E_{\max }$. The electron distribution at a time $t$ can be written (Pacholczyk 1970) as

$$
N(E, t)=\left\{\begin{array}{ll}
N_{0} E^{-\gamma}\left(1-\frac{E}{E_{\max }}\right)^{\gamma-2} & E_{\min }<E<E_{\max } \\
0 & E>E_{\max }
\end{array},\right.
$$

where

$$
E_{\min }=m_{e} c^{2}
$$

and $N_{0}$ is the normalization of the original distribution (Chevalier 1998) and is related to the equipartition factor:

$$
N_{0}=\frac{\tilde{\alpha}(\gamma-2) B^{2} E_{\min }^{\gamma-2}}{8 \pi} .
$$

\section{CALCULATING THE RADIO SPECTRUM}

To obtain the emission coefficient $\left(\epsilon_{\nu}\right)$ and absorption coefficient $\left(\kappa_{v}\right)$ using the modified electron population, we use the equations for $\epsilon_{v}$ and $\kappa_{v}$ from Pacholczyk (1970) as

$$
\begin{aligned}
& \epsilon_{v}=c_{3} H \sin \theta \int_{E_{\min }}^{\infty} N(E) F(x) d E, \\
& \kappa_{\nu}=-\frac{c^{2}}{2 v^{2}} c_{3} H \sin \theta \int_{E_{\min }}^{\infty} E^{2} \frac{d}{d E}\left(\frac{N(E)}{E^{2}}\right) F(x) d E,
\end{aligned}
$$

where

$$
x=\frac{v}{v_{c}}
$$

$$
\begin{gathered}
v_{c}=c_{1} H \sin \theta E^{2}, \\
F(x)=x \int_{x}^{\infty} K_{5 / 3}(z) d z .
\end{gathered}
$$

We can get the emission and absorption coefficients by substituting Equation (23) into Equations (26) and (27). We can write $E$ as a function of $x$ using $v_{c}=c_{1} H \sin \theta E^{2}$ as

$$
\begin{aligned}
E & =\frac{A}{\sqrt{x}}, \\
d E & =-\frac{1}{2} x^{-3 / 2} A d x,
\end{aligned}
$$

where

$$
A \equiv\left(\frac{v}{c_{1} H \sin \theta}\right)^{1 / 2} .
$$

The equations for the emission and absorption coefficients after substitution become

$$
\begin{aligned}
& \epsilon_{v}=-\frac{1}{2} c_{3} N_{0}\left(\frac{v}{c_{1}}\right) A^{-(\gamma+1)} I_{0}, \\
& \kappa_{v}=-\frac{c^{2}}{4 v^{2}} c_{3} N_{0}\left(\frac{v}{c_{1}}\right) A^{-\gamma}\left(A^{-2} I_{1}+A^{-1} I_{2}\right) .
\end{aligned}
$$

The integrals in the above formulae are as follows:

$$
\begin{aligned}
& I_{0}=\int_{x_{1}}^{x_{2}} x^{(\gamma-3) / 2} g(x)^{\gamma-2} F(x) d x, \\
& I_{1}=(\gamma+2) \int_{x_{1}}^{x_{2}} x^{(\gamma-2) / 2} g(x)^{\gamma-2} F(x) d x, \\
& I_{2}=\frac{(\gamma-2)}{E_{\max }} \int_{x_{1}}^{x_{2}} x^{(\gamma-3) / 2} g(x)^{\gamma-3} F(x) d x,
\end{aligned}
$$

where

$$
g(x)=\left(1-\frac{A x^{-1 / 2}}{E_{\max }}\right) .
$$

The limits of integration are given by

$$
\begin{aligned}
& x_{1}=\left(\frac{A}{E_{\text {min }}}\right)^{2}, \\
& x_{2}=\left(\frac{A}{E_{\max }}\right)^{2} .
\end{aligned}
$$

The source function is defined as

$$
S_{v}=\frac{\epsilon_{v}}{\kappa_{v}} .
$$

For our case, the source function becomes

$$
S_{v}=\frac{2 v^{2}}{c^{2}} \frac{A I_{0}}{\left(I_{1}+A I_{2}\right)} .
$$

The radiative transfer problem can be easily solved for the case of a planar emission region of thickness $s$,

$$
\pi R^{2} s=f \frac{4 \pi}{3} R^{3}
$$


where $f$ is the filling fraction; we use $f=0.5$ in our calculation. The radiative transfer equation is

$$
\frac{d I_{v}}{d \tau_{v}}=I_{v}-S_{v}
$$

It can be integrated simply in case of an homogeneous emission region from 0 to $s$ as

$$
I_{\nu}(s)=I_{\nu}(0) e^{-\tau_{\nu}(s, 0)}+\int_{0}^{s} \kappa_{\nu} S_{\nu} e^{-\tau_{\nu}\left(s, s^{\prime}\right)} d s^{\prime} .
$$

Since there is no incident radiation at $s=0, I_{v}(0)=0$, and the solution becomes

$$
I_{\nu}\left(\tau_{v}\right)=S_{v}\left(1-e^{-\tau_{v}}\right)
$$

where the optical depth $\tau_{v}$ is defined as

$$
\tau_{\nu}=\int_{0}^{s} \kappa_{v} d s=s \kappa_{\nu} .
$$

The flux can be calculated by integrating $I_{v}$ over the solid angle $\Omega$ :

$$
F_{\nu}=\int I_{\nu} d \Omega=S_{\nu}\left(1-e^{-\tau_{\nu}}\right) \Omega
$$

The integrals for the emission and absorption coefficients are evaluated numerically to obtain the radio light curves. The effect of FFA (Chevalier 1998) can be included as

$$
F_{\nu}=S_{\nu} \Omega\left(1-e^{-\tau_{v}}\right) \times \exp \left\{-\left(\frac{t}{t_{\mathrm{ff}}}\right)^{-3}\left(\frac{v}{v_{1}}\right)^{-2.1}\right\}
$$

where $t_{\mathrm{ff}}$ is the time at which the optical depth to FFA becomes unity at frequency $v_{1}$. In the calculation, we have used $v_{1}=3 \mathrm{GHz}$.

\section{RESULTS OF MODELING THE RADIO OBSERVATIONS}

Using the model described above we compute the radio fluxes and fit them to the observations as follows.

1. For a given explosion date $\left(t_{\mathrm{ex}}\right)$, fit the $3 \mathrm{GHz}$ radio light curve with an SSA model to obtain $m, F_{p}$, and $t_{p}$.

2. Calculate the radius $\left(R_{p}\right)$ and magnetic field $\left(B_{p}\right)$ estimates.

3 . Use $R_{p}, B_{p}$, and $t_{p}$ in the cooling model (Model-3 and Model-4) to obtain the best fit values of $t_{\mathrm{ff}}, t_{\mathrm{acc}}$, and $\log _{10}(\tilde{\alpha})$ based on $\chi^{2}$ minimization.

4. In computing Model-3 and Model-4, we use the optical light curve properly referenced according to the explosion date.

5. Compare models for different values of the explosion date.

Using the above procedure, we calculate best fit parameters by minimizing $\chi_{v}^{2}$ over the 3 -dimensional parameter space using Model-4 (Table 2). We use the $S(3.0 \mathrm{GHz}), C(5.0 \mathrm{GHz}), X$ $(8.5 \mathrm{GHz})$, and $K(21.0 \mathrm{GHz})$ band data for fitting purposes. The $K a(32.0 \mathrm{GHz})$ band observations are consistent with its light curve computed from the parameters obtained from fitting the other frequencies. The resolution of the grid is 0.05 along the $\log _{10}(\tilde{\alpha})$ axis, 0.5 days along the $t_{\mathrm{ff}}$ axis, and 0.025 days along

\begin{tabular}{|c|c|c|c|}
\hline Model & Properties & Parameters & $\chi_{v}^{2}$ \\
\hline \multirow[t]{2}{*}{ Model-1 } & $\begin{array}{c}\text { SSA } \\
\text { No Cooling }\end{array}$ & $\begin{array}{c}m, t_{\mathrm{ff}}, f_{p}, t_{p}, \gamma \\
m=1.1^{\mathrm{a}} \\
f_{p}(5 \mathrm{GHz})=0.52 \mathrm{mJy} \\
t_{p}(5 \mathrm{GHz})=29.28 \text { day } \\
\gamma=3.2\end{array}$ & 10.05 \\
\hline & & Inconsistent with $m \lesssim 1$ & \\
\hline \multirow[t]{2}{*}{ Model-2 } & $\begin{array}{c}\text { SSA+FFA } \\
\text { No Cooling }\end{array}$ & $\begin{array}{c}m, f_{p}, t_{p}, \gamma, t_{\mathrm{ff}} \\
m=1.1^{\mathrm{a}} \\
f_{p}(5 \mathrm{GHz})=0.61 \mathrm{mJy} \\
t_{p}(5 \mathrm{GHz})=23.64 \text { day } \\
\gamma=3.1 \\
t_{\mathrm{ff}}(5 \mathrm{GHz})=15.84 \text { day }\end{array}$ & 7.43 \\
\hline & & Inconsistent with $m \lesssim 1$ & \\
\hline Model- $3^{b}$ & SSA+Cooling & $\begin{array}{c}\tilde{t}_{\mathrm{acc}}, \tilde{\alpha} \\
m=0.97^{\mathrm{c}} \\
\gamma=3.1 \text { (fixed) } \\
\tilde{t}_{\mathrm{acc}}=0.55_{-0.15}^{+0.20} \text { day } \\
\log _{10} \tilde{\alpha}=2.70_{-0.70}^{+0.65}\end{array}$ & 7.45 \\
\hline Model- $4^{b}$ & SSA+FFA+Cooling & $\begin{array}{c}t_{\mathrm{ff}}, \tilde{t}_{\mathrm{acc}}, \tilde{\alpha} \\
m=0.97^{\mathrm{c}} \\
\gamma=3.1 \text { (fixed) } \\
t_{\mathrm{ff}}(3 \mathrm{GHz})=18.5_{-0.5}^{+0.5} \text { day } \\
\tilde{t}_{\mathrm{acc}}=0.53_{-0.18}^{+0.23} \text { day } \\
\log _{10} \tilde{\alpha}=2.04_{-0.6}^{+0.8}\end{array}$ & 6.50 \\
\hline
\end{tabular}
the $\tilde{t}_{\text {acc }}$ axis. For Compton cooling to be dominant, we need $\tilde{\alpha}>1.0$, therefore the region below $\log _{10}(\tilde{\alpha}) \underset{\tilde{t}}{<0.0}$ is rejected. We obtain best fit values of $t_{\mathrm{ff}}=18.5$ days, $\tilde{t}_{\mathrm{acc}}=0.53$ days,
Table 2

Radio-emission Modeling Results of SN 2012aw

Notes.

a Based on fit to the multiband radio data and explosion date 2012 March 16.10 UT.

${ }^{\mathrm{b}}$ For these models, we use the radius and magnetic field determined using the $3 \mathrm{GHz}$ light curve. Refer to Equations (7) and (14).

${ }^{\mathrm{c}}$ Based on fitting the $3 \mathrm{GHz}$ radio light curve.

and $\tilde{\alpha}=1.12 \times 10^{2}$ for the parameters. The $\chi_{v}^{2}$ corresponding to these parameter values is 6.5 . The contour plot visualizing the $\log _{10} \tilde{\alpha}-\tilde{t}_{\mathrm{acc}}$ space is shown in Figure 8 . The levels marked in the contour plot are separated by $\sim 0.2$. Because of the weak dependence of observed quantities on $\tilde{\alpha}$, it is not very strongly constrained by the radio observations alone. The values of the fitted parameters are reported in Table 2.

Another estimate of $\tilde{\alpha}$ can be obtained by using the observed $\mathrm{X}$-ray luminosity as the upper limit of the inverse-Compton contribution to the X-ray luminosity: ${ }^{13}$

$$
L_{\mathrm{obs}}^{X}=L_{\mathrm{IC}}^{X}+L_{\text {Thermal }}^{X} \Rightarrow L_{\mathrm{obs}}^{X} \gtrsim L_{\mathrm{IC}}^{X} .
$$

Using the expression for $E\left(d L_{\mathrm{IC}}^{X} / d E\right)$ from Chakraborti et al. (2012) and integrating it over the energy range $0.2 \mathrm{keV}$ to $2.0 \mathrm{keV}$, we get

$$
\begin{aligned}
8.8 & \times 10^{36} \gamma_{\min } S_{\star} \tilde{\alpha}^{11 / 19} V_{s 4}\left(\frac{L_{\mathrm{bol}}(t)}{10^{42} \mathrm{erg} \mathrm{s}^{-1}}\right) \\
& \times\left(\frac{t}{10 \text { days }}\right)^{-1} \lesssim L_{\mathrm{obs}}^{X}
\end{aligned}
$$

where $\gamma_{\min }$ is the minimum Lorentz factor of electrons, and $S_{\star}$ is the radio emission measure given by Equation (14) of

13 The X-ray luminosity equations assume that the circumstellar medium is formed by winds with constant parameters. 


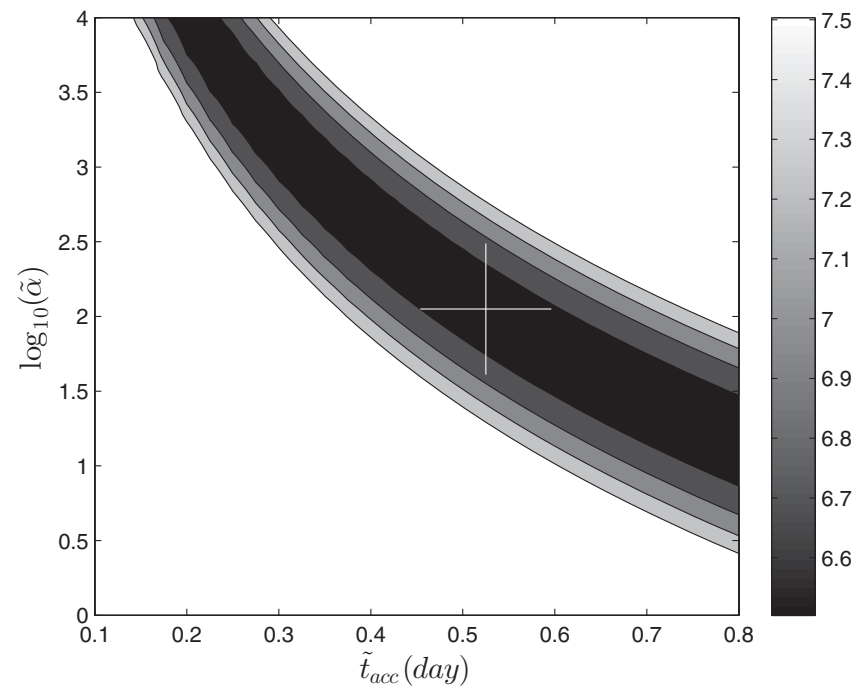

Figure 8. Calculated $\chi_{v}^{2}$ as a function of $\tilde{t}_{\mathrm{acc}}$ and equipartition factor $\left(\log _{10} \tilde{\alpha}\right)$ for $t_{\mathrm{ff}}=18.5$ days using Model-4 (refer to Table 2) with the explosion date 2012 March 15.3 UT. The cross represents the best fit values of $\tilde{t}_{\text {acc }}$ and $\tilde{\alpha}$ with $\chi_{v}^{2} \sim 6.50$. The grayscale shows the value of $\chi_{v}^{2}$ and the corresponding contours. The levels marked in the contour plot are separated by $\sim 0.2$. For Compton cooling to be dominant, we need $\tilde{\alpha}>1.0$, therefore the region below $\log _{10}(\tilde{\alpha})<0.0$ is rejected. The best fit parameters of this model are given in Table 2 .

Chakraborti et al. (2012),

$$
\begin{aligned}
S_{\star}= & 1.0\left(\frac{f}{0.5}\right)^{-8 / 19}\left(\frac{F_{\nu p}}{\mathrm{mJy}}\right)^{-4 / 19}\left(\frac{D}{\mathrm{Mpc}}\right)^{-8 / 19} \\
& \times\left(\frac{v}{5 \mathrm{GHz}}\right)^{2}\left(\frac{t}{10 \text { days }}\right)^{2} .
\end{aligned}
$$

Using $f=0.5$, we get $S_{\star}=3.97$ for SN 2012aw. The value of $L_{\mathrm{obs}}^{X}$ at an age of $\sim 5.6$ days is taken from Immler \& Brown (2012). Substituting $S_{\star}, V_{s 4} \sim R 0 / t 0$, and $L_{\text {bol }}=1.7 \times 10^{42}$ into Equation (54) gives

$$
\tilde{\alpha} \sim 0.35_{-0.24}^{+1.14} \times 10^{2} .
$$

This value smaller than the value of $\tilde{\alpha}$ that gives the best fit to the radio data, but both these values are consistent with each other within the error limits (see Table 2). Chandra observed the field of SN 2012aw on 2012 April 11. We analyzed the data and determined an X-ray luminosity of $(6.0 \pm 1.4) \times 10^{37} \mathrm{erg} \mathrm{s}^{-1} \mathrm{keV}^{-1}$ at $1.0 \mathrm{keV}$. This implies an $\tilde{\alpha} \sim 0.22_{-0.13}^{+0.45} \times 10^{2}$.

We note that the magnetic field and relativistic electrons are away from the equipartition regime. The value of $t_{\mathrm{ff}}$ can be used to get the $\dot{M} / v_{w}$ by inverting Equation (4) of Chevalier et al. (2006):

$$
\frac{\dot{M}_{-6}}{v_{w 1}} \approx\left(\frac{t_{\mathrm{ff}} T_{\mathrm{cs} 5}^{1 / 2} V_{s 4}}{6}\right)^{3 / 2}\left(\frac{v}{8.46 \mathrm{GHz}}\right)
$$

Using $V_{s} \sim R_{0} / t_{0}, T_{\mathrm{cs} 5} \sim 1.0$, and $t_{\mathrm{ff}}=18.5$ days at $v_{1}=3 \mathrm{GHz}$, we get

$$
\dot{M}_{-6} / v_{w 1} \sim 1.9
$$

The calculated radio light curves for the best fit parameters are shown in Figure 9. Using our model, we are able to explain the early-time data at high frequency.

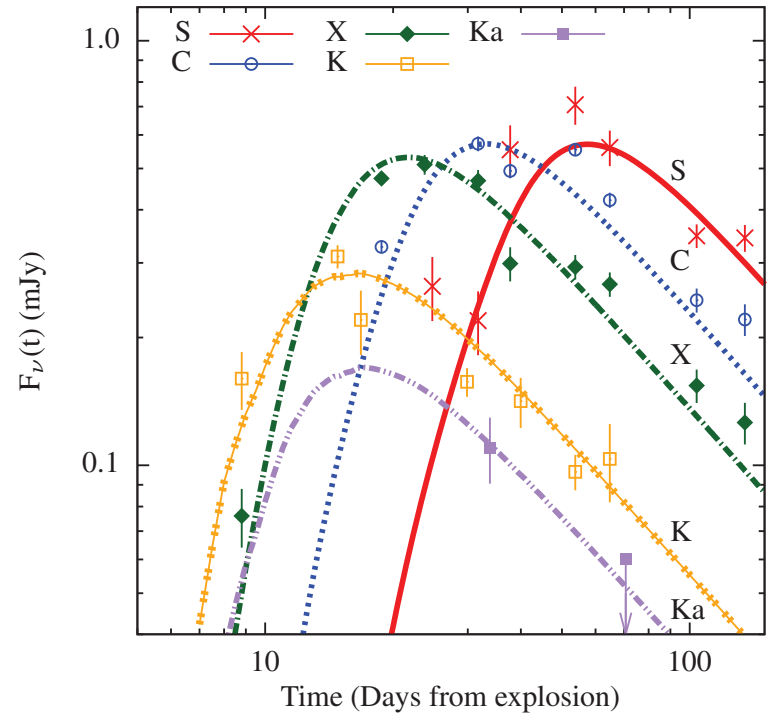

Figure 9. Radio observations of SN 2012aw and computed light curves based on Model-4 (Table 2). The parameters of the plotted model are: $t_{\mathrm{ff}}=18.5$ days, $\tilde{t}_{\text {acc }}=0.53$ days, and equipartition factor $\tilde{\alpha} \sim 1.12 \times 10^{2}$. The bands are as follows: $S(3.0 \mathrm{GHz}), C(5.0 \mathrm{GHz}), X(8.5 \mathrm{GHz}), K(21.0 \mathrm{GHz})$, and $K a$ $(32.0 \mathrm{GHz})$.

(A color version of this figure is available in the online journal.)

Table 3

Model Fits with Respect to the Fiducial Date of Explosion for Model-4 ${ }^{\mathrm{a}}$

\begin{tabular}{lccccc}
\hline \hline Explosion Date & $\chi_{v}^{2}$ & $m^{b}$ & $\begin{array}{c}t_{\mathrm{ff}} \\
\text { (day) }\end{array}$ & $\begin{array}{c}\tilde{t}_{\mathrm{acc}} \\
\text { (day) }\end{array}$ & $\log _{10} \tilde{\alpha}$ \\
\hline 2012 Mar 15.30 & 6.50 & 0.97 & 18.5 & 0.53 & 2.04 \\
2012 Mar 16.10 & 6.76 & 0.98 & 17.0 & 0.90 & 0.69 \\
2012 Mar 16.90 & 7.16 & 0.99 & 15.0 & 0.70 & 1.00 \\
\hline
\end{tabular}

Notes.

a The earliest detection of the SN (Fagotti et al. 2012) was on 2012 March 16.9 UT, whereas the last reported nondetection (Poznanski et al. 2012) was on 2012 March 15.3 UT.

${ }^{\mathrm{b}}$ Based on fitting the $3 \mathrm{GHz}$ radio light curve.

We also model the effect of varying the explosion date, $t_{\mathrm{ex}}$, since there is a time difference of 1.6 days between the last nondetection (Poznanski et al. 2012) and the first optical detection of the SN (Fagotti et al. 2012). The explosion date affects the calculation of the radio flux, especially at high frequencies, since the relativistic electrons experience different radiation environments due to the change of the density of UVOIR photons at the radiosphere. We calculate radio fluxes due to synchrotron emission by electrons for different explosion dates and fit the fluxes to the observed radio data. The results are summarized in Table 3 for Model-4 (refer to Table 2). The best fit is obtained for a $t_{\mathrm{ex}}$ of 2012 March 15.3.

\section{CONCLUSIONS AND DISCUSSION}

We have reported the radio observations of SN 2012aw, which has already been studied well in the optical and UV bands. Our observations spanning 184 days make it one of the best observed Type II-P radio SNe. We find that the spectral-index values are smaller than the values expected for the optically thin regime. We interpret this as a signature of electron cooling at a young age. Specifically, we find that inverse Compton cooling dominates over the synchrotron cooling process in the case of 
SN 2012aw. Although Chevalier et al. (2006) had predicted the effect of electron cooling on radio light curves, this is the first unambiguous evidence of cooling of relativistic electrons in a young SN due to inverse Compton scattering of lowenergy photons. We consider the effects of Compton cooling in order to self-consistently model the high-frequency radio emission. We fit the radio data to the model and estimate its parameters. We find that radiating plasma is away from equipartition $\left(\tilde{\alpha} \sim 1.12 \times 10^{2}\right)$ and relativistic electrons carry a greater fraction of the thermal energy compared to the postshock magnetic field. A similar result has been noted in the case of SN 2011dh (a Type IIb SN) by Horesh et al. (2013), for which $\tilde{\alpha} \sim 10^{3}$, which implies $\epsilon_{e} \gg \epsilon_{B}$ - the energy density in relativistic electrons exceeds the energy density in the magnetic field (Soderberg et al. 2012 have noted a value of $\tilde{\alpha} \sim 30$ for the case of SN 2011dh). The case of SN 1993J (another Type IIb) is in contrast to SN 2011dh, as in the former case Chandra et al. (2004) and Fransson \& Björnsson (1998) noted that the equipartition factor is $\sim 10^{-4}\left(\epsilon_{e} \ll \epsilon_{B}\right.$, the energy density in the magnetic field exceeds the energy density in relativistic electrons). We determine the value of $\dot{M} \sim 1.9 \times 10^{-6} M_{\odot} \mathrm{yr}^{-1}$, and it is consistent with the empirically estimated mass-loss rate for red giant progenitors of Type II-P SNe (Reimers 1977; de Jager et al. 1988). To investigate phenomena associated with electron cooling, observations of radio-bright $\mathrm{SNe}$ at young ages in high-frequency bands using ALMA and/or CARMA will be needed, as has been done in the case of SN 2011dh by Horesh et al. (2013) and Soderberg et al. (2012). Good, quality, early X-ray observations by Swift and/or Chandra are crucial to get stringent limits on the equipartition factor (including independent estimates on $\epsilon_{e}$ and $\epsilon_{B}$ ) and the contribution of thermal emission to the X-ray flux, as has been done in the case of SN 2004dj by Chakraborti et al. (2012).

Initial observation of SN 2012aw was requested under Joint Chandra-NRAO Cycle-13 ToO proposal (Proposal ID No. 13500809) on Type II-P SNe. A.R. thanks the Department of Physics at the West Virginia University for their hospitality during proposal development. We thank the anonymous reviewer for comments, queries, and suggestions, which significantly helped us in improving the manuscript. We acknowledge the support of TIFR 12th Five Year Plan (Project No: 12P-0261). We thank the Director Karl G. Jansky VLA for granting us the observations under
Director's Discretionary Time. N.Y. wishes to acknowledge the support of CSIR-SPM fellowship (SPM-07/858(0057)/2009EMR-I).

\section{REFERENCES}

Arnett, W. D. 1980, ApJ, 237, 541

Bayless, A. J., Pritchard, T. A., Roming, P. W. A., et al. 2013, ApJL, 764, L13 Bersten, M. C., Benvenuto, O., \& Hamuy, M. 2011, ApJ, 729, 61

Björnsson, C.-I., \& Fransson, C. 2004, ApJ, 605, 823

Bose, S., Kumar, B., Sutaria, F., et al. 2013, MNRAS, 433, 1871

Chakraborti, S., Ray, A., Smith, R., et al. 2013, ApJ, 774, 30

Chakraborti, S., Yadav, N., Ray, A., et al. 2012, ApJ, 761, 100

Chandra, P., Ray, A., \& Bhatnagar, S. 2004, ApJ, 612, 974

Chevalier, R. A. 1982, ApJ, 259, 302

Chevalier, R. A. 1996, in ASP Conf. Ser. 93, Radio Emission from the Stars and the Sun, ed. A. R. Taylor \& J. M. Paredes (San Francisco, CA: ASP), 125

Chevalier, R. A. 1998, ApJ, 499, 810

Chevalier, R. A., \& Fransson, C. 1994, ApJ, 420, 268

Chevalier, R. A., Fransson, C., \& Nymark, T. K. 2006, ApJ, 641, 1029

Chugai, N. N. 1991, SvAL, 17, 210

Cornwell, T., \& Fomalont, E. B. 1989, in ASP Conf. Ser. 6, Synthesis Imaging in Radio Astronomy, ed. R. A. Perley, F. R. Schwab, \& A. H. Bridle (San Francisco, CA: ASP), 185

de Jager, C., Nieuwenhuijzen, H., \& van der Hucht, K. A. 1988, A\&AS, 72,259

Dopita, M. A., Cohen, M., Schwartz, R. D., \& Evans, R. 1984, ApJL, 287, L69

Fagotti, P., Dimai, A., Quadri, U., et al. 2012, CBET, 3054, 1

Falk, S. W., \& Arnett, W. D. 1977, ApJS, 33, 515

Fransson, C., \& Björnsson, C.-I. 1998, ApJ, 509, 861

Fraser, M., Maund, J. R., Smartt, S. J., et al. 2012, ApJL, 759, L13

Hamuy, M. 2003, ApJ, 582, 905

Horesh, A., Stockdale, C., Fox, D. B., et al. 2013, MNRAS, 436, 1258

Immler, S., \& Brown, P. J. 2012, ATel, 3995, 1

Kochanek, C. S., Khan, R., \& Dai, X. 2012, ApJ, 759, 20

Litvinova, I. I., \& Nadezhin, D. K. 1983, Ap\&SS, 89, 89

Munari, U., Henden, A., Belligoli, R., et al. 2013, NewA, 20, 30

Pacholczyk, A. G. 1970, Series of Books in Astronomy and Astrophysics (San Francisco, CA: Freeman)

Poole, T. S., Breeveld, A. A., Page, M. J., et al. 2008, MNRAS, 383, 627

Popov, D. V. 1993, ApJ, 414, 712

Poznanski, D., Nugent, P. E., Ofek, E. O., Gal-Yam, A., \& Kasliwal, M. M. 2012, ATel, 3996, 1

Reimers, D. 1977, A\&A, 61, 217

Rybicki, G. B., \& Lightman, A. P. 1986, in Radiative Processes in Astrophysics, ed. G. B. Rybicki \& A. P. Lightman (New York: Wiley), 400

Smartt, S. J. 2009, ARA\&A, 47, 63

Soderberg, A. M., Margutti, R., Zauderer, B. A., et al. 2012, ApJ, 752, 78

Stockdale, C. J., Ryder, S. D., Van Dyk, S. D., et al. 2012, ATel, 4012, 1

Van Dyk, S. D., Cenko, S. B., Poznanski, D., et al. 2012, ApJ, 756, 131

Yadav, N., Chakraborti, S., \& Ray, A. 2012, ATel, 4010, 1 